\title{
Quantum noise measurements in a continuous- wave laser-diode-pumped Nd:YAG saturated amplifier
}

\author{
Shally Saraf, Karel Urbanek, and Robert L. Byer \\ E. L. Ginzton Laboratory, Stanford University, Stanford, California 94305 \\ Peter J. King \\ LIGO Project, California Institute of Technology, Pasadena, California 94305
}

Received September 20, 2004

\begin{abstract}
We present measurements of the power noise due to optical amplification in a Nd:YAG free-space travelingwave amplifier as the amplifier transitions from the linear regime into the heavily saturated regime. The quantum noise behavior is demonstrated by saturating the gain of a $100-\mathrm{W}$ class zigzag slab amplifier with a high-power beam and measuring the power noise detected by a single-spatial-mode probe beam traversing the same optical path through the amplifier. (C) 2005 Optical Society of America

OCIS codes: $140.3480,140.3280,140.4480,270.2500,270.5290$.
\end{abstract}

High-power, $\mathrm{TEM}_{00}$-mode, low-noise lasers are required for the Laser Interferometer Gravitational Wave Observatory (LIGO). ${ }^{1}$ The LIGO interferometer is a highly sensitive instrument that is designed to detect strains of the order of $10^{-22}$ created by gravitational waves emanating from astronomical sources such as neutron stars and black hole binary inspirals. Since detector shot noise scales inversely with laser power, there is a strong incentive to scale the existing $10-\mathrm{W}$ diffraction-limited, singlefrequency LIGO laser source to the $200-\mathrm{W}$ level and beyond for improved sensitivity. Master oscillator power amplifier (MOPA) based systems are well suited for power scaling while preserving the spatial and temporal coherence of the master oscillator. ${ }^{2}$ However, MOPA configurations are noisy because of the power noise added by the unavoidable spontaneous emission into the spatial mode being amplified. Saturating an amplifier reduces the power noise while extracting most of the available gain. Therefore, in a MOPA configuration the initial amplifiers that are operating in the linear and weakly saturated regime will set the noise figure of the amplifier chain. Adding saturated amplifier stages with minimal noise figure degradation provides an approach for power scaling to meet the laser requirements for the LIGO.

In this study we confirm the noise performance of a free-space Nd:YAG optical amplifier in the nonlinear or saturated regime. Previous work at Stanford has demonstrated the noise performance of unsaturated amplifiers, ${ }^{3}$ where the quantum noise scales linearly with the power gain of the amplifier. Prior work on the noise performance of saturated amplifiers has focused on fiber amplifiers and has shown that the effect of gain saturation is to increase the signal-tonoise ratio relative to the case of an unsaturated amplifier. ${ }^{4}$ To our knowledge, we present the first quantitative measurement of the quantum noise of a free-space Nd:YAG optical amplifier as it transitions from the linear regime into the heavily saturated regime.

The starting point of noise analysis is the photon statistics master equation from the fundamental paper by Shimoda et al. ${ }^{5}$ describing the evolution of a photon stream in an amplifier based on the stochastic process of birth, death, and immigration ${ }^{6}$ :

$$
\frac{\mathrm{d} P_{n}}{\mathrm{~d} x}=a\left[n P_{n-1}-(n+1) P_{n}\right]+b\left[(n+1) P_{n+1}-n P_{n}\right],
$$

where $P_{n}$ is the probability of finding exactly $n$ photons in the system, $a=\sigma_{e} N_{2}$ and $b=\sigma_{a} N_{1}$, where $\boldsymbol{\sigma}_{e}, \boldsymbol{\sigma}_{a}$ are the emission and absorption cross sections and $N_{2}, N_{1}$ are the populations in the upper and lower lasing levels, respectively. The first and second moments are sufficient to quantify the noise introduced by the amplifier when the number of photons is very large and the input photon stream obeys Poisson statistics. In a fully inverted four-level laser system such as Nd:YAG, spontaneous-emission factor $f_{\mathrm{sp}}$ is only a function of transmission loss $\alpha$ in the gain medium and power gain $G$ as defined by $f_{\mathrm{sp}}=1$ $+[\alpha / \ln (G)]$. There is loss at the transmission window of the detector $\eta_{\mathrm{tr}}$ and imperfect quantum conversion at detector $\eta_{q}$; because they are Bernoulli random deletion processes, they preserve the photon statistics and result in an efficiency factor in the noise equation of $\eta=\eta_{\mathrm{tr}} \eta_{q}$. Solving Eq. (1) then yields the following expression for the noise power $\sigma_{d}^{2}$ at the detector for an unsaturated amplifier ${ }^{4}$

$$
\sigma_{d}^{2}=\sigma_{\text {shot }}^{2}\left[1+2 f_{\text {sp }} \eta(G-1)\right],
$$

where $G$ is the power gain of the amplifier and $\sigma_{\text {shot }}{ }^{2}$ is the shot-noise power of the amplifier output.

In a saturated amplifier the statistical coupling of the atomic and photon populations complicates computation of the moments. Decorrelation methods 
have been used to provide approximate solutions. ${ }^{7}$ However, in this experiment the saturating beam and the probe beam are separate and uncorrelated as explained below, and the saturated power gain for the probe may be written as $G_{\text {sat }}=\exp \left(g_{o} l / 1+s n_{\text {ext }}\right)$, where $g_{0} l$ is the small-signal gain, $n_{\text {ext }}$ is the uncorrelated saturating beam photon flux, and $s=1 / n_{\text {sat }}$ is defined as the saturation coefficient. ${ }^{4}$ The expression for the noise power $\sigma_{d}^{2}$ of the probe beam at the output of a saturated amplifier with saturated gain $G_{\text {sat }}$ is identical in form to the one derived above for the unsaturated amplifier case and is given by

$$
{\sigma_{d}}^{2}=\sigma_{\text {shot }}^{2}\left[1+2 f_{\mathrm{sp}} \eta\left(G_{\mathrm{sat}}-1\right)\right] .
$$

Figure 1 shows the experimental setup. A shot-noiselimited probe beam is generated from a monolitihic, single-frequency, single-spatial-mode nonplanar ring oscillator (NPRO). ${ }^{8}$ The NPRO is locked to a monolithic, high-finesse, impedance-matched three-mirror Fabry-Perot ring cavity also referred to as a mode cleaner ${ }^{9}$ operating in $s$ polarization and at resonance using the Pound-Drever-Hall rf technique with an electro-optic modulator (EOM). ${ }^{10}$ The cavity has a finesse of 4400 , a free spectral range of $714 \mathrm{MHz}$, and a $3-\mathrm{dB}$ cutoff frequency of $87 \mathrm{kHz}$. The high-finesse mode cleaner reduces the power noise and non$\mathrm{TEM}_{00}$ modes of the NPRO output through temporal and spatial filtering and results in a shot-noise-limited probe beam at the input to the amplifier.

The high-power beam generated in a MOPA chain is also locked to an identical Fabry-Perot cavity but with a much lower finesse of approximately 50 operating in $p$ polarization. The two cavities have identical construction except for the reflectivity of the input and output couplers and consequently support identical $\mathrm{TEM}_{00}$ eigenmodes. This translates into identical spot sizes of the beams at the output of the cavities and permits the beams to overlap with a high degree of precision. The temperature of the Nd:YAG crystal in the NPRO is tuned to ensure a frequency

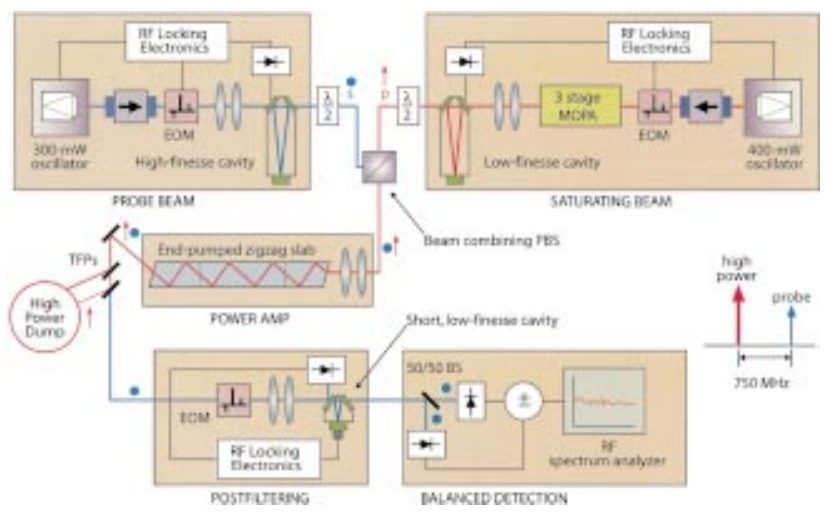

Fig. 1. Layout of the quantum noise measuring system of a Nd:YAG free-space saturated amplifier. The orthogonally polarized probe and high-power saturating beams are locked to identical Fabry-Perot cavities, overlapped, and sent through an end-pumped Nd:YAG slab amplifier. The beams are then separated and the probe is sent to a balanced detection system to measure quantum noise. offset of approximately $750 \mathrm{MHz}$ between the locked probe and the high-power saturating beams. This frequency offset is much less than the $200-\mathrm{GHz}$ fluorescence bandwidth of Nd:YAG; therefore the overlapped probe and high-power beams have the same power gain. However, the frequency offset permits separation of the beams after the amplification process with a third Fabry-Perot cavity with a large free spectral range as described below.

The two beams are combined on a polarizing beam splitter equidistant from the two Fabry-Perot cavities, thus ensuring identical beam spot sizes. The overlapped high-power beam and the probe beam are then sent through a power amplifier and experience the same power gain. The two beams are uncorrelated, since they are derived from two sources that have no spatial or temporal correlation and also have a frequency offset of $750 \mathrm{MHz}$. Additionally, the master oscillators have Faraday isolators and the finesse of the cavities provides further rejection of any injection-seeding feedback between the two lasers.

The power amplifier in the system is a $100-\mathrm{W}$ class end-pumped Nd:YAG zigzag slab. The slab is symmetrically pumped with a pair of $808-\mathrm{nm}$ fibercoupled laser diode arrays at power levels of up to $175 \mathrm{~W}$ per fiber. The high-power saturating beam is varied from $0 \mathrm{~W}$, which corresponds to a linear unsaturated amplifier, to $27 \mathrm{~W}$, in which case the amplifier is heavily saturated with an input intensity of almost nine times the saturation intensity for Nd:YAG. The spot size in the 6.4-cm-long amplifier with a $3.3-\mathrm{cm}$ long doped region is $285 \mu \mathrm{m}$.

The amplified saturating high-power beam and probe beam are separated after the end-pumped slab amplifier by a pair of thin-film polarizers (TFPs). The polarizers alone do not provide rejection of the power coupled from the saturating high-power beam into the probe beam because of the thermally induced birefringence in the slab at high pump powers. However, further temporal and spatial postfiltering is performed by locking the probe beam to the resonance of a short low-finesse mode cleaner operating in $s$ polarization. The postfiltering Fabry-Perot cavity has a finesse of 125 and a free spectral range of $3 \mathrm{GHz}$. The $750-\mathrm{MHz}$ frequency offset between the probe and high-power beams results in an additional $30-\mathrm{dB}$ rejection of the high-power saturating beam in both polarizations. This postfiltering is critical to the success of the experiment because it avoids detector saturation by rejecting leaked power from the high-power beam.

The output of the short low-finesse mode cleaner is then sent through a 50:50 beam splitter (BS) into a balanced detector. The narrow passband of the Fabry-Perot cavity transmits the probe and completely rejects amplified spontaneous emission outside the passband and 808-nm pump light from the amplifier. The difference output of the balanced detector is the uncorrelated shot-noise reference level for the signal and the sum output gives the total power noise on the signal. The balanced detector outputs are measured with a HP70000 rf spectrum analyzer over the frequency range $6.25-15.625 \mathrm{MHz}$ to 
measure the quantum noise added to the signal through amplification. This range is chosen based on the frequency response of the photodetection system.

The spontaneous-emission factor $f_{\mathrm{sp}}$ is calculated on the basis of the measured transmission loss of $1.25 \% / \mathrm{cm}$ through the end-pumped slab and the measured power gain of the probe. The transmission efficiency of the photodetector window is measured at $95 \%$, and the quantum efficiency of the detectors is measured to be $86 \%$. Figure 2 shows a plot of the measured quantum noise versus power gain of the probe beam at several saturating power levels. The fit over a wide dynamic range of power gain to the quantum noise equation (3) is excellent in both cases of unsaturated and saturated gain in the amplifier.

Figure 3 shows in detail the amplifier noise behavior as it transitions from the unsaturated to the heavily saturated regime. The dashed horizontal lines locate the experimental points from Fig. 3(a) on Fig. 3(b). Figure 3(a) shows the measured power noise of the probe relative to the shot-noise limit for the 90-mA photocurrent for an unsaturated amplifier in the absence of a high-power beam. Figure 3(b) shows the power noise on the probe beam as the high-power beam saturates the gain of the amplifier.

The theoretical curves in Figs. 3(a) and 3(b) are plots of Eqs. (2) and (3), respectively, based on the measured power gain and loss in the slab and the measured efficiencies of the photodetection process. Additionally, the power extraction efficiency of the amplifier versus the high-power beam intensity is plotted in Fig. 3(b) based on the extracted power and the available power in the slab at the highest pump power of $350 \mathrm{~W}$. At an input power of $27 \mathrm{~W}$, which corresponds to $I / I_{\text {sat }}$ of 8.6 , the extraction efficiency of the amplifier is $\sim 93 \%$, and the quantum noise is 2.8 times the shot-noise limit at $90-\mathrm{mA}$ photocurrent. Figure 3 clearly shows that as the power is increased the power extraction efficiency from the amplifier approaches 1 , and the quantum noise level is reduced toward the shot-noise limit set by losses in the amplifier as predicted by theory. The present LIGO laser is a $10-\mathrm{W}$ MOPA that is followed by a premode cleaner and a 12-m suspended mode cleaner. These mode cleaners, along with the 4-km-long interferometer, filter laser noise and result in shot-noise-limited

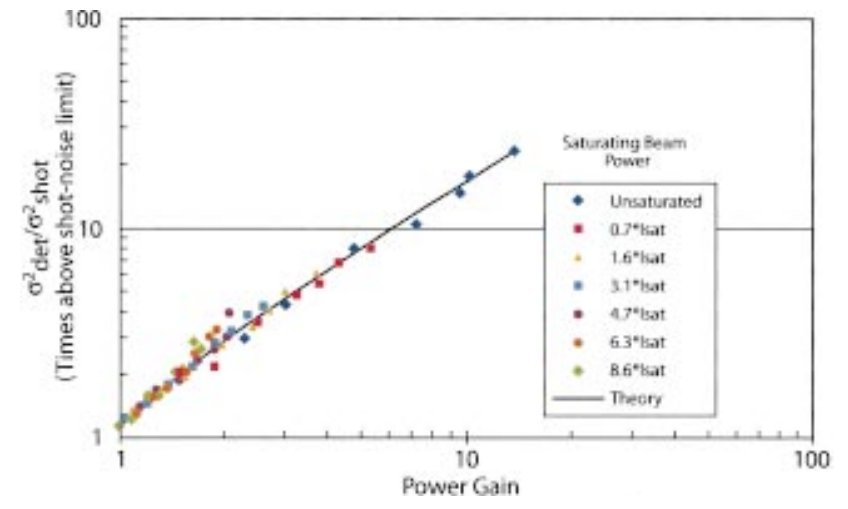

Fig. 2. Plot of quantum noise versus power gain for different input intensities of the high-power saturating beam. The theory curve is a plot of the quantum noise Eq. (2).

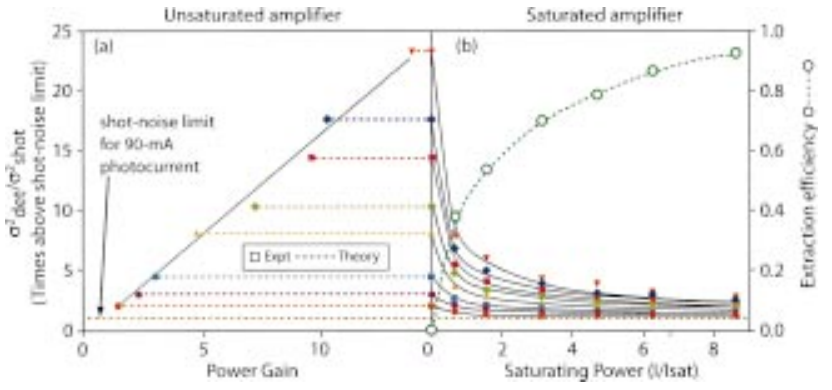

Fig. 3. (a) Linear scaling of quantum noise of an unsaturated amplifier as the pump power is increased. (b) Quantum noise curves as the gain is saturated down with a highpower beam overlapping the probe beam. The dashed curve shows the extraction efficiency of the power amplifier as it transitions to deep saturation.

operation at the $\mathrm{rf}$ modulation frequencies. ${ }^{9}$ The filtering action of these cavities allows scaling of the existing 10-W MOPA to higher powers by the addition of saturated amplifier stages while preserving shotnoise-limited operation.

In summary we have measured the high-frequency noise of a free-space linear and saturated optical amplifier. We have demonstrated that a MOPA system can be scaled to higher powers by adding saturated amplifier stages with a minimum noise penalty and with greater than $90 \%$ power extraction efficiency. We have demonstrated the use of Fabry-Perot cavities to generate a shot-noise-limited probe beam and to precision overlap and separate beams with a high contrast in power levels.

This work was supported in part by National Science Foundation grants PHY-0140297 and PHY9210038 and in part by the U.S. Army Research Office under ARO grants DAAD19-02-1-0184. S. Saraf's e-mail address is saraf@stanford.edu.

\section{References}

1. B. C. Barish and R. Weiss, Phys. Today 52(10), 44 (1999).

2. W. Wiechmann, T. J. Kane, D. Haserot, F. Adams, G. Truong, and J. D. Kmetec, in Conference on Lasers and Electro-Optics, Vol. 6 of 1998 OSA Technical Digest Series (Optical Society of America, Washington, D.C., 1998), p. 432.

3. W. M. Tulloch, T. S. Rutherford, E. H. Huntington, R. Ewart, C. C. Harb, B. Willke, E. K. Gustafson, M. M. Fejer, R. L. Byer, S. Rowan, and J. Hough, Opt. Lett. 23, 1852 (1998).

4. E. Desuvivre, Erbium-Doped Fiber Amplifiers (Wiley, New York, 1994).

5. K. Shimoda, H. Takahasi, and C. H. Townes, J. Phys. Soc. Jpn. 12, 687 (1957).

6. C. W. Helstrom, Probability and Stochastic Processes for Engineers (Macmillan, New York, 1998).

7. G. Oliver and C. Bendjaballah, Phys. Rev. A 22, 630 (1980).

8. T. J. Kane and R. L. Byer, Opt. Lett. 10, 65 (1985).

9. B. Willke, N. Uehara, E. K. Gustafson, R. L. Byer, P. King, S. Seel, and R. L. Savage, Jr., Opt. Lett. 23, 1704 (1998).

10. R. W. P. Drever, J. L. Hall, F. V. Kawalski, J. Hough, G. M. Ford, A. J. Munley, and H. Ward, Appl. Phys. B 31, 97 (1983). 